\title{
Human Figure Animation: A Historical Perspective
}

\author{
Stephania Loizidou Himona \\ Frederick University \\ Cyprus
}

\author{
Yiorgos Chrysanthou \\ University of Cyprus \\ Cyprus
}

\author{
Andreas Loizides \\ PA College \\ Cyprus
}

\section{Keywords}

Computer Animation, Human Figure Animation, Simulation, Locomotion, Survey.

\begin{abstract}
Computer Animation, has advanced significantly as well as spectacularly over the last two-to-three decades. The interesting field has innovated tremendously moving from first-generation i.e. purely geometric models where motion was developed using kinematic techniques, to secondgeneration i.e. physical-based models (direct versus inverse dynamics and hybrid systems), towards the development of the third-generation i.e. autonomous behaviour through learning and perception. The simulation and animation of a variety of real-world objects appear with stunning realism. However, the main issue here is not on what we have achieved but rather, based on what we have achieved, what is to follow. The emerging results are expected immensely by researchers in the field, worldwide. The paper delivers a survey on human figure animation and in particular a classification framework of the work done on the control of locomotion.
\end{abstract}

\section{Introduction}

Computer Animation of human figure locomotion is becoming more and more popular in the recent years with virtual humans being used extensively in three-dimensional simulation environments. Such computer animation can be used in a wide range of applications, such as computer games, film industry, multimedia, training, education, medicine, virtual reality, architecture, security etc.

Although a number of different techniques (kinematics, dynamics, biomechanics, robotics, etc) have been used for producing realistic or believable motion, in many ways computer animation of human figures still remains a challenging problem. Walking as a motion is a very challenging control task due to its dynamically unstable nature, which requires continual balance to prevent the human character not only from falling over but also from reaching poses that are out of joint limits. In addition to that, people are very skilled at perceiving the subtle details of human motion and the viewer can easily recognise as well as reason when a motion appears artificial and unnatural.

An "efficient" human locomotion system should be able to model different types of motions and to account for dif- ferent ways that a person could walk, not to mention different moods, conditions and other sociological as well as psychological states that affect the individual's gait style.

That is, the content (refers to the nature of the action in the movement) as well as the style (denotes the particular way that the action is performed) are the two major factors that should be taken into consideration when dealing with the perception of the human motion. These two factors should be in interaction with each other but at the same time in separation. For example, a system that can synthesise stylistic variations of a given action would be a useful tool. Such a system that applies user-specified styles to prerecorded motion sequences (as well as extrapolating styles not included in the original data), for a small database, is proposed in (Torresani et al., 2007). Another system that learns to control planar walking gaits of a given style is analysed in (Sharon and van de Panne, 2005).

This paper outlines a comparative study of previous work done in the area of motion control in computer animation focusing on the different methods available together with the solution that each method assisted towards the development of human figure locomotion, i.e. the complex mechanism of human motion, according to the referred application. It aims to be used as a study guide, by covering the relevant literature as well as providing a classification framework. Once the current solutions are identified the paper explores areas of future developments.

\section{Advances in Human Figure Animation}

Human figure animation is an interesting as well as a challenging subset of Computer Graphics. It engaged people of different disciplines from the early days. Long before movie cameras were invented, Eadweard Muybridge's photographic sequences of animal (using a still camera he photographed a horse running to prove that when it gallops there are times when it has all four feet off the ground, (Muybridge, 1955)) as well as anthropomorphic locomotion became a point of reference for many researchers.

Studying the activities of human beings as well as their behaviour under different circumstances has become a focal point that has been addressed not only by computer scientists (the use of synthetic actors in 3D simulation environments, e.g. simulations of crowds and human behaviours in virtual reality applications), recently, but originally by psychologists, sociologists, medical practitioners, physicians and many others needless to mention the appli- 
cations in the film industry. However, because there is not an easy way for people in one field to know what people in the other fields are working on and thus achieving, there is usually little or no interdisciplinary exchange of ideas and as a consequence results are slowly progressing.

\section{Human Locomotion}

Human locomotion is an important activity as well as an interesting problem closely related to man's everyday life. It is a complicated issue, since the articulations contained in the human figure have enormous complexity. The model has a large number of (rotary) joints each with several degrees of freedom (DOF) and the interaction between these joints is controlled by the muscles. Besides, there exist a large number of variations in the movement of individuals, so that exact specification and control of their movements is, in most cases, practically impossible.

The fact that human movement patterns are extremely familiar, (few movements are as familiar and recognizable as human walking and running (Hodgins, 1998)), and the viewer can easily recognize as well as reason when a motion appears artificial and unnatural, complicates the matter further. That is, people are skilled at detecting subtleties in human motion (Hodgins et al., 1998) which makes the animation of human figures an important, but at the same time, a difficult problem in computer animation.

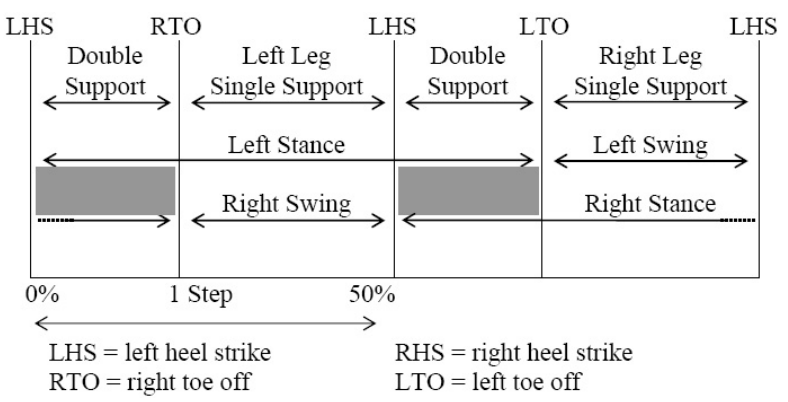

Figure 1: Locomotion cycle for bipedal walking

For simplification, walking is broken up into two phases; the stance and the swing phase, and these are further broken up into sub-phases. Figure 1, describes the locomotion cycle for bipedal walking from biomechanics (sets the start of the walking cycle on the left heel strike event) as described in (Loizidou, 1992). Biomechanics principles to represent walking motion on slopes and around curves has been used by Sun and Metaxas (2001).

\section{Human Figure Animation - Classification Overview}

An early classification (Terzopoulos, 1999) tried to distinguish between the different levels of computer animation, and in particular human figure animation, from its beginning towards its then state; geometric versus physically based models. It is believed that a further category has been created since then, namely, the beginning of autonomous behaviour through learning and perception.

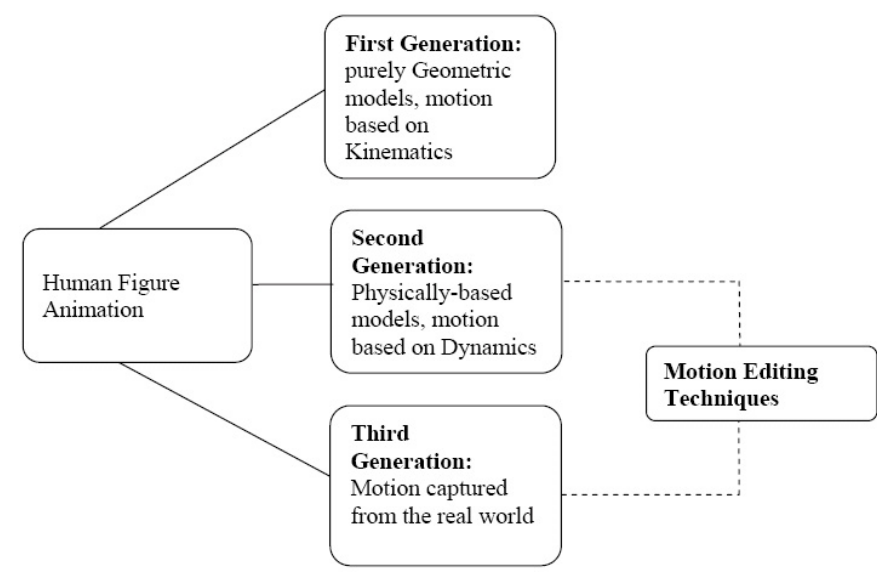

Figure 2: Categorization of human figure animation

Figure 2, illustrates this categorization. The next section describes these three levels, outlining the evolution that progressed from purely kinematic "knowledge-based" methods (1980's) towards approaches that incorporate dynamic simulations to generate motions (1990's) and even towards interactive blending and further tuning of synthetic or captured motion (1997 onwards); see also (Multon et al., 1999). The aim is not on emphasizing on the historical perspective of this classification but rather on describing the intrinsic advantages and/or limitations of each one of them.

\section{First Generation}

During the first-generation i.e. purely geometric models, motion was developed using kinematic techniques (direct and inverse kinematics, relied in biomechanical knowledge) which basically helped in keyframing (Zeltzer, 1983), (Lasseter, 1987), (Wilhelms, 1987), (Bruderlin and Calvert, 1996), (Hodgins, 1998), when trying to achieve expressiveness and believable behaviour. Keyframing is basically the technique whereby the animators have to explicitly specify the definition of the key values of the character's DOF at specific instances called the "key-frames". Interpolation of the values of the DOF is then used (e.g. cubic spline techniques) to compute the in-between frames (Perlin, 1995), (Kovar and Gleicher, 2003), (Safonova and Hodgins, 2005).

However, the end result, regardless of the fact that it was easy to produce (low computational cost), was not at all realistic and had nothing to do with its proximity to real life (Wilhelms, 1987). That is, the major drawback of the method concerned the lack of motion realism and therefore researchers had to combine it with appropriate normalization (Boulic et al., 1990), correction techniques to minimize sliding (Boulic, 2003) and foot constraints with inverse kinematics.

\section{Second Generation}

Moving towards the second-generation i.e. physical-based models versus inverse dynamics (Vasilonikolidakis and 
Clapworthy, 1991) as well as hybrid systems (Loizidou and Clapworthy, 1993)), the motion was improved tremendously, however this was done at the expense of complicated physical laws of motion equations (e.g. Newton's laws, Lagrange mechanics, Featherstone's equations of motion etc) with quite a number of DOF even for a simple representation of e.g. the human body. To simulate human bodies is difficult due to the fact that the human motion is controlled by internal torques applied to the joints. The main problem here is how to specify forces and torques, which actually move the human figure in a way, which the animator might desire (Le et al., 2003).

Although the results have been realistic not much has been done about accounting for feelings, attitude, emotions and behaviour in the body animation. The motion has to be purely directed by the animator but then again the movement had to be predefined with no interesting variations from one animation sequence to the next. The main concern at that point was the realism of the movement with the minimum calculations possible as well as the fastest of the particular application.

Controlling the motion at a high level was basically the main problem. There was not an easy, convenient or even a possible way to specify the form of motion using flexible set of movement commands that could generate a variety of motions. High-level descriptions of commands like "walk happily" or "walk vigorously" where these commands would be translated automatically into the corresponding parametric descriptions of the motion was still under investigation.

To overcome some of the problems stated above, researchers have tried to combine methods from the first and second generations. As an indication, Wilhelms (Wilhelms, 1987) used a hybrid kinematics/dynamics approach where controlling functions, representing positions over time, had to be entered explicitly before starting the simulation, without knowing intuitively what forces or torques would be necessary to produce the desired motion. Girard (Girard, 1987), (Girard, 1991) and Girard \& Maciejewski (Girard and Maciejewski, 1985), also used a hybrid kinematics/dynamics algorithm and therefore all the problems associated with the kinematics approach were again present when attempting to control articulated figures. A similar approach has been adopted by Bruderlin and Calvert (Bruderlin and Calvert, 1989) and is discussed by Calvert (Calvert, 1991). Isaacs and Cohen (Isaacs and Cohen, 1987) presented another combination of kinematics and inverse dynamics specifications.

Furthermore, Hahn (Hahn, 1988) presented a method that merged kinematics and dynamics for articulated rigid bodies. He used kinematics to control joint (internal) trajectories and dynamics to model the effects of limb motion (external analysis) and external forces and torques on the body as a whole.

As it can be observed there was no way that using any one of these techniques alone was possible to achieve the required results. Therefore, a combination of two or more of these techniques was necessary according to the specified application for which they had been developed for.

\section{Third Generation}

The conventional classification for the third generation involves autonomous behaviour through learning and perception. However, other researchers allow for some other categorization, with the allowance of an extra sub-level which could be introduced between the 2 nd and 3rd generation, i.e. motion interpolation which is basically the combination of pre-existing motion data, e.g. through motion capture (Badler et al., 1993), (Hodgins and Wooten, 1995), (Thalmann and Musse, 2007) (which is basically considered as the first stage in the process of producing exploitable animation data (Menache, 2000)), or even motion by example (Arikan and Forsyth, 2002), (Kovar and Gleicher, 2003), (Lerner et al., 2007); so that new motions can be produced.

Motion capture uses magnetic systems or optical (visionbased sensors) systems to record the actions of a real human character in 3D. This data (usually from a database pool) is then used to animate the virtual character. However, it is interesting to note that keeping the size of the database manageable as well as determining when a database has sufficient variety of motions is still an open area requiring further work. The acquisition is not free of problems, magnetic systems suffer from noisy data, while optical systems have problems related to occlusion.

It is rarely the case that captured motions can be used straight away and be effective in every respect. The real and its corresponding mapped simulated character should have exactly the same number of joints, the same segment proportions and the same skinning for the animation to fulfil the plain re-use of the captured motions (the size and the quality of the database resulting from motion capture is the key to success; the database must be large enough so as good transitions can be found (Hodgins and Wooten, 1995) plus accountability of contacts with the environment). That is, it is interesting to look for genericity (any size and any proportions characters) of the versatile walk engine (Boulic et al., 2004). That is why motion editing techniques (blending and warping) need always be applied for modifying and varying captured motions (see Gleicher (Gleicher, 2001) for a survey; as well as Gleicher et al. (Kovar et al., 2002) for a technique on retrieving the best-fitting animation using "point clouds" to estimate the distance of postures).

The lack of flow between different animation clips is also equally important in order to avoid unnatural-looking frozen postures between motions. A system for real-time virtual human idle motion synthesis for the body is proposed (Egges et al., 2004). This is based on motion capture (i.e. existing clips). As a basis for the motion generation, a Principal Component Analysis (PCA) is used to determine dependencies between joints in the various postures. PCA also allows for easy blending and fitting of motions 


\begin{tabular}{|c|l|l|l|}
\hline Level & Method & Characteristics & Applications \\
\hline 1 & $\begin{array}{l}\text { Geometric models } \\
\text { (Kinematic techniques) }\end{array}$ & $\begin{array}{l}\text { - Low computational costs, O(n) } \\
\text { - Lack of motion realism }\end{array}$ & $\begin{array}{l}\text { Virtual Reality, Movie Production, } \\
\text { Video Games (pre-scripted motion) }\end{array}$ \\
\hline 2 & $\begin{array}{l}\text { Physically-based models } \\
\text { (Dynamic techniques) }\end{array}$ & $\begin{array}{l}\text { - Realistic motion } \\
\text { - High computational costs, } \mathrm{O}\left(n^{3}\right)\end{array}$ & $\begin{array}{l}\text { Virtual Reality, Art, } \\
\text { Biomechanical Simulations }\end{array}$ \\
\hline 3 & $\begin{array}{l}\text { Motion Editing } \\
\text { (Interpolation methods) }\end{array}$ & $\begin{array}{l}\text { - Low computational costs, O(n) } \\
\text { - Large volume of motion database } \\
\text { - Realistic trajectories } \\
\text { - Hard interactive control }\end{array}$ & $\begin{array}{l}\text { Video Games, } \\
\text { Movie Productions, Art, } \\
\text { Virtual Reality }\end{array}$ \\
\hline 4 & $\begin{array}{l}\text { Autonomous behaviours } \\
\text { (Learning and Percep- } \\
\text { tion) }\end{array}$ & $\begin{array}{l}\text { - Automatic simulation code generation } \\
\text { - High computational costs, } \mathrm{O}\left(n^{3}\right) \\
\text { - Accounts for dynamic effects }\end{array}$ & $\begin{array}{l}\text { Virtual Reality, Art, Movies, } \\
\text { Behaviour simulation, } \\
\text { Biomedical simulations }\end{array}$ \\
\hline
\end{tabular}

Table 1: Levels of human figure animation

resulting in flexible and realistic animations in real time. The whole approach permits the specification of different databases for different persons, which allows for a personalized aspect in the animations.

Motion warping and motion blending are the two major families of techniques for modifying the captured motion. Motion warping (takes well-known trajectories, e.g. keyframes and modifies them in order to change the motion, thus creating smooth transitions without discontinuity (Witkin and Popovic, 1995), (Torresani et al., 2007)) and motion blending (avoids the jerkiness in the transitions by using a database of captured motions and interpolation techniques in order to generate new motions) are almost always necessary for preserving the fine details of the motion (Witkin and Popovic, 1995), (Bruderlin and Williams, 1995). Furthermore, constraint-based motion editing techniques (Gleicher, 1998), (Lee and Shin, 1999), (Popović and Witkin, 1999), (Rose et al., 1996) are also necessary to avoid e.g. foot sliding.

\section{Analysis}

Table 1, summarizes the levels of the models used for computer animation, in particular for human figure animation, giving the advantages and the disadvantages of each together with the application areas that each one is best suitable for.

Each model has its own number of advantages and disadvantages. As an indication, Table 2 compares the main two animation methods that have prevailed in recent years (dynamic techniques versus motion capture methods). From Table 2 one can conclude that dynamic techniques offer more advantages compared to motion capture methods, however this does not restrict researchers to use the latter (growing popularity) due to the fact that it produces realistic results, one of the most (if not the most) valued characteristic (motion capture data is the most common technique in commercial animation because many of the subtle details of human motion are naturally present in the data (Lee et al., 2002)) regardless of its price. In addition, motion capture databases are gradually being built and made available to the wider audience. However, the problems associated with motion capture, see also Table 2, prevent it from being the ideal solution for all applications.

It is thus understood that using one model alone would not solve the specific problem and it is further believed that a combination of the different models from the different levels could solve particular problems and as a result enhance in the creation of autonomous virtual characters capable to react realistically to their environment. Combining, for example, motion capture and dynamic simulation is an emerging area under exploration, in order to retain the advantages of each while avoiding their disadvantages (Zordan and Hodgins, 2002). That is, information about human motion contained in motion capture data coupled with the physical realism provided by simulation, could provide controllable and reactive human motion (Arikan et al., 2005), (Yin et al., 2007), (Sok et al., 2007).

Although the peak of its usage was reported at SIGGRAPH 2007 (6 papers by (Sok et al., 2007), (Treuille et al., 2007), (Cooper et al., 2007), (Chai and Hodgins, 2007), (Safonova and Hodgins, 2007), (Yin et al., 2007)) the idea of combining more than one of the techniques for human figure animation is not new. It originates from the innovative idea of Zordan and Hodgins that use a forward dynamics and a tracking controller to follow human motion data i.e. dynamics with motion capture (Zordan and Hodgins, 1999). At that point, they did not report it as an area of broad potential; their work was basically motivated from the three previous, similar ideas of (i) (Bruderlin and Calvert, 1989) that use simple simulation and kinematics (biomechanical heuristics), (ii) (Witkin and Popovic, 1995) that use motion capture with interpolating joint angles (blending between two sequences of joint angles to create new joint trajectories), (iii) (Rose et al., 1996) that use inverse dynamics and torque minimisation (inverse dynamics between fixed motion sequences).

Furthemore, at that time motion capture equipment was of limited capabilities (limited availability and quality) and on top of that developing robust dynamic controllers that produce natural looking motion was a very hard problem. 


\begin{tabular}{|c|c|}
\hline $\begin{array}{l}\text { Dynamic techniques } \\
\text { (force-based simulation of movement) }\end{array}$ & $\begin{array}{l}\text { Motion Capture techniques } \\
\text { (pre-recorded movements) }\end{array}$ \\
\hline $\begin{array}{l}\text { 1. Lack of naturalness of the capture movement data } \\
\text { (simulating robots) }\end{array}$ & $\begin{array}{l}\text { 1. They capture also the naturalness of the movements } \\
\text { (humans) }\end{array}$ \\
\hline 2. Variant movement data (always producing new data) & $\begin{array}{l}\text { 2. Invariant motion (monotone) sequences (repeti- } \\
\text { tions/reuse); limited to real-world characters }\end{array}$ \\
\hline 3. High computing requirements (too expensive) & $\begin{array}{l}\text { 3. No computations to calculate the movement data (pre- } \\
\text { recorded data) }\end{array}$ \\
\hline $\begin{array}{l}\text { 4. No mapping needed. No retargeting (new data). Re- } \\
\text { finement is required }\end{array}$ & $\begin{array}{l}\text { 4. Perfect mapping/retargeting/adaptation is required } \\
\text { (analyze/modify extract captured data: qualitative anal- } \\
\text { ysis) }\end{array}$ \\
\hline $\begin{array}{l}\text { 5. Physically correct forces, not realistic; i.e. physical } \\
\text { validity often sacrificed for performance }\end{array}$ & $\begin{array}{l}\text { 5. No changing of the fine structure of the original cap- } \\
\text { ture data (no forces taken into consideration) }\end{array}$ \\
\hline 6. The only stage required & 6. It can only be used as the first stage \\
\hline $\begin{array}{l}\text { 7. Flexible and Interactive motion control of complex } \\
\text { mechanical systems. Real-time programming }\end{array}$ & 7. No interactivity \\
\hline $\begin{array}{l}\text { 8. Very complicated tasks: specification and solution } \\
\text { of the equations of motion, dedicated control techniques } \\
\text { (extensive mathematics and physics) }\end{array}$ & $\begin{array}{l}\text { 8. Provides motion data for all DOF at a very high level } \\
\text { of detail }\end{array}$ \\
\hline 9. No other equipment necessary & 9. Motion capture systems are very expensive \\
\hline 10. "Easy to use" animation system & $\begin{array}{l}\text { 10. Cables connection restricts actor's range of motion, } \\
\text { therefore difficult to record certain movements }\end{array}$ \\
\hline $\begin{array}{l}\text { 11. High degree of reusability, due to motions that can be } \\
\text { generated automatically }\end{array}$ & $\begin{array}{l}\text { 11. Repetitions of capture sessions (until the desired mo- } \\
\text { tion is achieve) can be costly and labour intensive. There- } \\
\text { fore editing \& processing the motion capture data after it } \\
\text { has been collected in order to make it re-usable }\end{array}$ \\
\hline
\end{tabular}

Table 2: Dynamic versus Motion Capture methods - (shading indicates an advantage)

Therefore, nobody could really predict then the scale of its today's (and possibly tomorrow's) usage not to mention the capabilities of such a combination.

The objective would be to capture the subtle details of realistic human-like motion while at the same time maintaining the physical realism of the movement. Ideally, virtual worlds with enhanced physics would bring greater realism and expression. The emphasis should be on bringing the real and virtual worlds closer together by bridging their associated strengths.

One such method, although still simple and at its infancy, is discussed by (Zordan et al., 2005), whereby it combines dynamic simulations together with a motion captured database. This system selects the appropriate clip from the motion capture repertoire (the search engine) and once an interaction has happened, computes new motion based on physical conditions (the joint-torque controller). Dynamics is thus used only for a short time burst (e.g. during the effects of a collision) and after that the character finds an available motion from the capture repository, therefore, minimising the computational bottleneck associated with dynamic techniques.

A more simple idea is to combine interpolation with motion capture (Abe et al., 2004), (Safonova and Hodgins, 2007). In such systems, the desired motion is represented as an interpolation of two time-scaled paths through a mo- tion graph. That is, it retains the natural transitions of motion graphs and the ability to synthesize physically realistic variations provided by interpolation. However, the quality of the results depends largely on the size (Treuille et al., 2007) (only a few minutes (Safonova and Hodgins, 2007)) as well as the quality of the motion database used to construct the motion graph (the appropriateness of the database (Chai and Hodgins, 2007), whereby once again motion capture data is used as a motion prior while matching constraints are specified by the user), together with the "good", and/or automatic, connectivity of the motion graph (Ikemoto et al., 2007). Furthermore, the idea of evaluating motion graphs is outlined by Reitsma and Pollard (Reitsma and Pollard, 2004), in order to identify how much motion data should be included in the motion graph, in addition to assessing the character's capabilities while comparing motion datasets for the improvement of the motion graph.

Chiu et al. also use motion graphs as a means for reusing motion capture data by building transitions between motion segments and forming a tightly connected network of movement (Chiu et al., 2007).

Cooper et al. provide some other combinations of motion capture data (Cooper et al., 2007). They produce the character's motion by interpolating the motion capture and they proceed to use active learning for improving the quality and the responsiveness of the motion controller. The 
active learning is used for identifying the regions of the control space that do not perform well, and thus improving them. However, the system focuses on a specific model for motion control with a limited number of tasks. Another interesting idea uses motion capture (a motion engine) together with a controller (a control policy) which selects appropriate sequences of clips to achieve some goal quickly and naturally (Treuille et al., 2007). Once again this technique requires large amounts of pre-captured motion data, to produce highly varied controllers.

Another interesting work is by Shapiro et al., whereby once again the combination of motion capture is reported (Shapiro et al., 2003). However, they claim that this is the first time were the combination of dynamic and kinematic control is done in a uniform fashion, thus producing complex motion and interaction for animated characters.

However, more recently research has progressed towards the development of the evolutionary autonomous behaviour through learning and perception. That is, the evolution is to create self animating characters that learn to react appropriately to their perceived environment, thus, reducing much of the workload from the animators. This coincides with the interesting field of artificial intelligence whereby characters are free to react like living creatures. For example, the interaction between a pedestrian and his/her environment is still a focal point of interest in this field.

Researchers are working towards the development of autonomous virtual humans in a proper environment model which can analyze the particularity of each situation/environment that they appear each time, make decisions as well as behave naturally accordingly (Devillers et al., 2002), (Pelechano et al., 2007). Progressing through different levels of abstraction, these models amalgamate proper modelling together with motion however the main issue is the inclusion of perception and behaviour (Shao and Terzopoulos, 2005).

It is well understood that behaviour animation has its origins strongly connected to crowd simulation, (Thalmann and Musse, 2007). Reynolds work on flocks of birds (large groups of entities called boids) and fish (fishes) could be considered as the first example in the field of behavioural animation whereby we could notice that group behaviour is resulting from the interaction between the individual behaviours of its group members (Reynolds, 1987). Although his work was based on three simple rules it led to the emergence of a much more complex flocking behaviour (Reynolds, 1999).

Similar, and even more complex, work by Tu and Terzopoulos created a realistically rich environment inhabited by artificial fishes (Tu and Terzopoulos, 1994). Simulations of large crowd structures and crowd behaviours, in real time, using three levels of autonomy were proposed by the work of Musse and Thalmann (Musse and Thalmann, 2001). Work on simulating crowds of virtual humans while focusing on the individual agents instead of the groups, where the behaviour model was composed of three levels, was also proposed by Ulicny and Thalmann (Ulicny and Thalmann, 2002). Individual agents behaviour planning is additionally discussed in (Lau and Kuffner, 2005), (Chiu et al., 2007), whereby the automatic generation of realistic motions using a planning algorithm, that performs a global search in a finite-state machine (FSM) of behaviours of organized motion captured clips, is described (the motion capture technique will be discussed later in this section).

Furthermore, HiDAC (High-Density Autonomous Crowds) can be tuned to simulate realistically different types of crowds using psychological, physiological and geometrical rules combined with physical forces (Pelechano et al., 2007). These allow for individual heterogeneous behaviours while accounting for perception, reaction to static and dynamic objects, appropriate individual collision avoidance and collision response. Though, the way of controlling the properties and behaviours of individual agents in the crowd (instead of lower level parameters, i.e. expanding this personality model) remains still a challenge.

As a consequence, researchers in the area of computer graphics and animation of human figures should start thinking of possibilities which would begin from the very top level, and for overcoming specific problems of the certain level, to direct the model towards integrating lower levels. Thus, for example, motion editing techniques would very much be aided by the use of dynamic constraints which in turn would much be aided by kinematic correction models. It is strongly believed that such a combination of techniques would solve and overcome most, if not all, of the problems associated with each model alone. The possibilities are endless.

\section{Conclusion}

This paper classifies the techniques that have been used extensively during the last two-to-three decades, for the simulation of human locomotion. The aim has been to survey the different methods, specifying explicitly from where they have started towards where they are directed, that is, to provide a taxonomy of the recent techniques together with their solutions as well as chronological reasons. The relationships (comparing and contrasting) between a number of state-of-the-art methods for animating human-like figures is explored together with a unifying view of several techniques and their applications as well as to give insights to the unexplored regions of the animation world.

It is well understood that things are progressive; however a lot has still to be done before we are in the contented situation whereby we can look at something played by a computer (in particular human figures) and not to be apparent as to whether it is actually real or computer simulated; not to mention the possibility of controlling it in real time (i.e. enhancing interactively in terms of higher-level actions for obtaining adaptive behaviour). 


\section{Future Directions}

Future work could focus on combining the modelled methods which would solve disadvantages associated with a particular method if used alone. Another possibility would be to extend the animation sequences, together with the animated agents that appear on individual frames, so as to create more interesting, complex scenes and therefore to manage the natural variations overtime for believable crowds. Furthermore, the need for automatically creating large number of believable gaits for a crowd simulation is also a close necessity.

Evaluation of human locomotion is another area of possible research. It could be done qualitatively (e.g. through real and simulated video images) as well as quantitatively (e.g. through simulated and biomechanical data). Although not of particular concern in this paper's contents, the resulting perception of the human motion is affected by the geometric model (Hodgins and Wooten, 1995). There exists an enormous variety of models and rendering styles and it therefore becomes essential to adopt a unifying set of models for presenting results and ease in comparing them, e.g. using statistical data. For this, several forms of evaluation to test the motion, e.g. video comparison, biomechanical data or online Turing test could be used.

\section{REFERENCES}

Abe, Y., Liu, C., and Popovic, Z. (2004). Momentum-based parameterization of dynamic character motion. ACM SIGGRAPH, Eurographics Symposium on Computer Animation, pages $173-182$.

Arikan, O. and Forsyth, D. (2002). Interactive motion generation from examples. ACM Transactions on Graphics, 21(3):483490.

Arikan, O., Forsyth, D., and O'Brien, J. (2005). Pushing people around. Proceedings of the 2005 ACM SIGGRAPH/Eurographics symposium on Computer animation, pages 59-66.

Badler, N., Hollick, M., and Granieri, J. (1993). Real-Time Control of a Virtual Human Using Minimal Sensors. Presence, 2(1):82-86.

Boulic, R. (2003). Re-Appraising Procedural Motion Modeling: Illustration on a Walk Engine. Eurographics Workshop on Virtual Environments, Zurich.

Boulic, R., Magnenat-Thalmann, N., and Thalmann, D. (1990). A Global Human Walking Model with Real-Time Kinematic Personification. The Visual Computer, 6(6):344-358.

Boulic, R., Ulicny, B., and Thalmann, D. (2004). Versatile Walk Engine. Journal of Game Development, 1(1):29-50.

Bruderlin, A. and Calvert, T. (1989). Goal-Directed, Dynamic Animation of Human Walking. Proceedings of the 16th Annual Conference on Computer Graphics and Interactive Techniques, pages 233-242.

Bruderlin, A. and Calvert, T. (1996). Knowledge-Driven, Interactive Animation of Human Running. Proceedings of the Conference on Graphics Interface, pages 213-221.

Bruderlin, A. and Williams, L. (1995). Motion Signal Processing. Proceedings of the 22nd Annual Conference on Computer Graphics and Interactive Techniques, pages 97-104.
Calvert, T. (1991). Composition of Realistic Animation Sequences for Multiple Human Figures. Morgan-Kaufmann Series In Computer Graphics And Geometric Modeling, pages $35-50$.

Chai, J. and Hodgins, J. (2007). Constrained-Based Optimisation Using a Statistical Dynamic Model. International Conference on Computer Graphics and Interactive Techniques.

Chiu, B., Zordan, V., and Wu, C. (2007). State-annotated motion graphs. Proceedings of the 2007 ACM symposium on Virtual reality software and technology, pages 73-76.

Cooper, S., Hertzmann, A., and Popović, Z. (2007). Active Learning for Real-Time Motion Controllers. International Conference on Computer Graphics and Interactive Techniques.

Devillers, F., Donikian, S., Lamarche, F., and Taille, J. (2002). A programming environment for behavioural animation. The Journal of Visualization and Computer Animation, 13(5):263274.

Egges, A., Molet, T., and Magnenat-Thalmann, N. (2004). Personalized Real-Time Idle Motion Synthesis. Computer Graphics and Applications, 12th Pacific Conference, pages 121-130.

Girard, M. (1987). Interactive Design of 3D Computer-Animated Legged Animal Motion. IEEE Computer Graphics and Applications, 7(6):39-51.

Girard, M. (1991). Constrained Optimization of Articulated Animal Movement in Computer Animation. Making Them Move: Mechanics, Control, and Animation of Articulated Figures.

Girard, M. and Maciejewski, A. (1985). Computational Modeling for the Computer Animation of Legged Figures. Proceedings of ACM SIGGRAPH, pages 263-270.

Gleicher, M. (1997). Motion Editing with Spacetime Constraints. Proceedings of the 1997 Symposium on Interactive 3D Graphics, pages 139-148.

Gleicher, M. (1998). Retargetting Motion to New Characters. Proceedings of the 25th Annual Conference on Computer Graphics and Interactive Techniques, pages 33-42.

Gleicher, M. (2001). Comparing Constraint-Based Motion Editing Methods. Graphical Models, 6(2):107-134.

Hahn, J. (1988). Realistic Animation of Rigid Bodies. Proceedings of the 15th Annual Conference on Computer Graphics and Interactive Techniques, pages 299-308.

Hodgins, J. (1998). Animating Human Motion. Scientific American, 278(3):46-51.

Hodgins, J., O'Brien, J., and Tumblin, J. (1998). Perception of Human Motion with Different Geometric Models. IEEE Transactions on Visualization and Computer Graphics, 4(4):307316.

Hodgins, J. and Wooten, W. (1995). Animating Human Athletes. ACM SIGGRAPH, 95:71-78.

Ikemoto, L., Arikan, O., and Forsyth, D. (2007). Quick Transitions with Cached Multi-Way Blends. Proceedings of the 2007 Symposium on Interactive $3 D$ Graphics and Games, pages $145-151$

Isaacs, P. and Cohen, M. (1987). Controlling Dynamic Simulation with Kinematic Constraints. Proceedings of the 14th Annual Conference on Computer Graphics and Interactive Techniques, pages 215-224.

Kovar, L. and Gleicher, M. (2003). Flexible Automatic Motion Blending with Registration Curves. Proceedings of the 2003 ACM SIGGRAPH/Eurographics Symposium on Computer Animation, pages 214-224.

Kovar, L., Gleicher, M., and Pighin, F. (2002). Motion Graphs. Proceedings of the 29th Annual Conference on Computer Graphics and Interactive Techniques, 21(3):473-482. 
Lasseter, J. (1987). Principles of Traditional Animation Applied to 3D Computer Animation. ACM SIGGRAPH Computer Graphics, 21(4):35-44.

Lau, M. and Kuffner, J. (2005). Behavior Planning for Character Animation. Proceedings of the 2005 ACM SIGGRAPH / Eurographics Symposium on Computer Animation, pages 271-280.

Le, D., Boulic, R., and Thalmann, D. (2003). Integrating Age Attributes to Virtual Human Locomotion. International Archieves of Photogrammy, Remove Sensing and Spatial Information Science, XXXIV-5.

Lee, J., Chai, J., Reitsma, P., Hodgins, J., and Pollard, N. (2002). Interactive Control of Avatars Animated with Human Motion Data. Proceedings of the 29th Annual Conference on Computer Graphics and Interactive Techniques, 21(3):491-500.

Lee, J. and Shin, S. (1999). A Hierarchical Approach to Interactive Motion Editing for Human-Like Figures. Proceedings of the 26th Annual Conference on Computer Graphics and Interactive Techniques, pages 39-48.

Lerner, A., Chrysanthou, Y., and Lischinski, D. (2007). Crowds by Example. Computer Graphics Forum, 26(3):655-664.

Loizidou, S. (1992). Dynamic Analysis of Anthropomorphic Manipulators in Computer Animation. University of North London.

Loizidou, S. and Clapworthy, G. (1993). Legged Locomotion Using HIDDS. Models and Techniques in Computer Animation, pages 257-269.

Menache, A. (2000). Understanding Motion Capture for Computer Animation and Video Games. Morgan Kaufmann.

Multon, F., France, L., Cani-Gascuel, M., and Debunne, G. (1999). Computer Animation of Human Walking: A Survey. The Journal of Visualization and Computer Animation, 10(1):39-54.

Musse, S. and Thalmann, D. (2001). Hierarchical Model for Real Time Simulation of Virtual Human Crowds. IEEE Transactions on Visualization and Computer Graphics, 7(2):152-164.

Muybridge, E. (1955). The Human Figure in Motion. Dover Publications.

Pelechano, N., Allbeck, J., and Badler, N. (2007). Controlling Individual Agents in High-Density Crowd Simulation. Proceedings of the 2007 ACM SIGGRAPH/Eurographics Symposium on Computer Animation, pages 99-108.

Perlin, K. (1995). Real time responsive animation with personality. IEEE Transactions on Visualization and Computer Graphics, 1(1):5-15.

Popović, Z. and Witkin, A. (1999). Physically based motion transformation. Proceedings of the 26th annual conference on Computer graphics and interactive techniques, pages 11-20.

Reitsma, P. and Pollard, N. (2004). Evaluating Motion Graphs for Character Navigation. Proceedings of the 2004 ACM SIGGRAPH / Eurographics Symposium on Computer Animation, pages $89-98$

Reynolds, C. (1987). Flocks, Herds and Schools: A Distributed Behavioral Model. ACM SIGGRAPH Computer Graphics, 21(4):25-34.

Reynolds, C. (1999). Steering Behaviors for Autonomous Characters. Game Developers Conference, 1999:763-782.

Rose, C., Guenter, B., Bodenheimer, B., and Cohen, M. (1996). Efficient generation of motion transitions using spacetime constraints. Proceedings of the 23rd annual conference on Computer graphics and interactive techniques, pages 147-154.

Safonova, A. and Hodgins, J. (2005). Analyzing the physical correctness of interpolated human motion. Proceedings of the 2005 ACM SIGGRAPH/Eurographics symposium on Com- puter animation, pages $171-180$.

Safonova, A. and Hodgins, J. (2007). Construction and Optimal Search of Interpolated Motion Graphs. International Conference on Computer Graphics and Interactive Techniques.

Shao, W. and Terzopoulos, D. (2005). Autonomous Pedestrians. Proceedings of the 2005 ACM SIGGRAPH/Eurographics Symposium on Computer Animation, pages 19-28.

Shapiro, A., Pighin, F., and Faloutsos, P. (2003). Hybrid control for interactive character animation. Computer Graphics and Applications, 2003. Proceedings. 11th Pacific Conference on, pages 455-461.

Sharon, D. and van de Panne, M. (2005). Synthesis of Controllers for Stylized Planar Bipedal Walking. Proceedings of the IEEE International Conference on Robotics and Automation, pages 2387-2392.

Sok, K., Kim, M., and Lee, J. (2007). Simulating Biped Behaviors from Human Motion Data. International Conference on Computer Graphics and Interactive Techniques.

Sun, H. and Metaxas, D. (2001). Automating Gait Animation. Proceedings of ACM SIGGRAPH 2001, pages 261-270.

Terzopoulos, D. (1999). Artificial Life for Computer Graphics. Communications of the ACM, 42(8):32-42.

Thalmann, D. and Musse, S. (2007). Crowd Simulation. Springer, 1 st edition.

Torresani, L., Hackney, P., and Bregler, C. (2007). Learning Motion Style Synthesis from Perceptual Observations. Proc. NIPS, 19.

Treuille, A., Lee, Y., and Popović, Z. (2007). Near-Optimal Character Animation with Continuous Control. International Conference on Computer Graphics and Interactive Techniques.

Tu, X. and Terzopoulos, D. (1994). Artificial Fishes: Physics, Locomotion, Perception, Behavior. Proceedings of the 21st Annual Conference on Computer Graphics and Interactive Techniques, pages 43-50.

Ulicny, B. and Thalmann, D. (2002). Towards Interactive RealTime Crowd Behavior Simulation. Computer Graphics Forum, 21(4):767-775.

Vasilonikolidakis, N. and Clapworthy, G. (1991). Design of Realistic Gaits for the Purpose of Animation. Computer Animation, 91:101-114

Wilhelms, J. (1987). Using dynamic analysis for realistic animation of articulated bodies. IEEE Computer Graphics and Applications, 7(6):12-27.

Witkin, A. and Popovic, Z. (1995). Motion Warping. Proceedings of the 22nd Annual Conference on Computer Graphics and Interactive Techniques, pages 105-108.

Yin, K., Loken, K., and van de Panne, M. (2007). SIMBICON Simple Biped Locomotion Control. International Conference on Computer Graphics and Interactive Techniques.

Zeltzer, D. (1983). Knowledge-Based Animation. ACM SIGGRAPH SIG ART. Workshop on Motion, pages 187-192.

Zordan, V. and Hodgins, J. (1999). Tracking and modifying upper-body human motion data with dynamic simulation. Computer Animation and Simulation99, 2:280-285.

Zordan, V. and Hodgins, J.K. (2002). Motion Capture-Driven Simulations that Hit and React. In Proceedings of the 2002 ACM SIGGRAPH / Eurographics Symposium on Computer Animation, pages 89-96. ACM Press.

Zordan, V., Majkowska, A., Chiu, B., and Fast, M. (2005). Dynamic response for motion capture animation. ACM Transactions on Graphics, 24(3):697-701. 